\title{
DIRICHLET FINITE BIHARMONIC FUNCTIONS ON THE POINCARÉ $N$-BALL
}

\author{
BY D. HADA, L. SARIO, AND C. WANG ${ }^{1}$ \\ Communicated by W. T. Martin, February 8, 1973
}

On a Riemannian manifold $R$, let $\Delta=d \delta+\delta d$ be the LaplaceBeltrami operator. By definition, a sufficiently smooth function $u$ on $R$ is harmonic (biharmonic) if $\Delta u=0\left(\Delta^{2} u=0\right)$. Denote by $D$ the class of those functions $f$ on $R$ for which $D(f)=\int_{R} d f \wedge * d f$ is well defined and finite.

By the Poincaré $N$-ball we mean the ball

$$
B_{\alpha}^{N}=\left\{x=\left(x^{1}, \ldots, x^{N}\right)|| x \mid<1\right\},
$$

$\alpha$ constant, endowed with the Poincaré-type metric

$$
d s_{\alpha}=\lambda(x)|d x|, \quad \lambda(x)=\left(1-|x|^{2}\right)^{\alpha} .
$$

The first purpose of this paper is to determine those values of the parameter $\alpha$ for which the class $H^{2} D\left(B_{\alpha}^{N}\right), N \geqq 3$, of Dirichlet finite nonharmonic biharmonic functions on $B_{\alpha}^{N}$ is nonvoid. In Sario-Wang [3] it was proved that $H^{2} D\left(B_{\alpha}^{N}\right) \neq \varnothing$ for $N=3$ if and only if $\alpha>-3 / 5$, and the question was raised whether the same is true for every $N$ if and only if $\alpha>-3 /(N+2)$. We show that this is indeed so if $3 \leqq N \leqq 6$. However, quite unexpectedly, for $N>6$ it turns out that $H^{2} D\left(B_{\alpha}^{N}\right) \neq \varnothing$ if and only if $\alpha \in(-3 /(N+2)$, $5 /(N-6))$.

The above result has interesting applications to the classification theory. Let $Q$ be the class of quasiharmonic functions $u$, defined by $\Delta u=1$, and denote by $Q D$ the subclass of Dirichlet finite functions in $Q$. The classes $O_{G}, O_{Q D}$, and $O_{H^{2} D}$ of Riemannian manifolds without Green's functions, $Q D$-functions, and $H^{2} D$-functions, respectively, have the following properties:

(i) For every $N$, the classes $O_{Q D}$ and $O_{H^{2} D}$ decompose the totality of Riemannian $N$-manifolds into three nonempty disjoint subclasses.

(ii) For every $N$, the class $O_{G}-O_{H^{2} D}$ is nonvoid.

(iii) For $N>6$, the classes $O_{G}$ and $O_{H^{2} D}$ decompose the totality of Riemannian $N$-manifolds into four nonempty disjoint subclasses.

(iv) The unit $N$-ball with the natural metric $\left(1-|x|^{2}\right)|d x|$ belongs to $O_{H^{2} D}$ if and only if $N>10$.

The proofs will appear in [1].

AMS (MOS) subject classifications (1970). Primary 31B30.

${ }^{1}$ This work was sponsored by the U.S. Army Research Office-Durham, Grant DA-ARO-D-31-124-71-G181, University of California, Los Angeles. 


\section{BIBLIOGRAPHY}

1. D. Hada, L. Sario and C. Wang, Dirichlet finite biharmonic functions on the Poincaré $N$-ball, J. Reine Angew. Math. (to appear).

2. L. Sario and C. Wang, Quasiharmonic functions on the Poincaré $N$-ball, Rend. Mat. (to appear).

3. - Existence of Dirichlet finite biharmonic functions on the Poincaré 3-ball, Pacific J. Math. (to appear).

Department of Mathematics, University of California, los Angeles, California 90024 